\title{
Cannabis exacerbation of bipolar disorder
}

\section{Background}

Cannabis-induced mood disorders have long been suspected by mental health practitioners, despite the lack of formal recognition by DSM IV. The diagnostic findings are further conflated by the existence of self-medicating behaviors that may accompany cannabis consumption. A literature search reveals an abundance of articles that explore the relationship between cannabis and psychosis with respect to emergent schizophrenia. However, cannabis exacerbation of bipolar disorder is also supported by a longitudinal study, independent research as well as anecdotal evidence.

\section{Objective}

In our literature review, we hope to differentiate factors that may contribute to the overall development of a mood disorder, in particular, bipolar disorder, as a function of cannabis use. Our review will place an emphasis on individuals that exclusively developed a mood disorder subsequent to cannabis use as opposed to self-medicating behaviors that may accompany a pre-existing or underlying bipolar condition. Lastly, we will examine the role of sleep as it relates to cannabis consumption and bipolar disorder.

According to Kaplan and Saddock's Concise Textbook of Clinical Psychiatry, hypomania may be expressed as a common side effect of cannabis intoxication. ${ }^{1}$ Thus, if we were to appropriately address causative issues of interest, the literature review must recognize a distinction between hypomania as an immediate side effect that corresponds with acute cannabis intoxication and a hypomanic mood state; a hypomanic mood state may be expressed within the context of an overarching, bipolar disorder that has developed as a function of chronic cannabis use. ${ }^{2}$

\section{Method}

A comprehensive search was conducted using Pubmed, Google Scholar and various journal databases to assess the role of cannabis with respect to bipolar disorder exacerbation, as well as sleep. The literature review encompasses 12 articles between the years 1982 and 2015. We discuss pertinent case studies, as well as information extrapolated from longitudinal population-based studies and other literature reviews.

\section{Discussion}

A case report examined an acute psychotic episode secondary to cannabis abuse that eventually precipitated the onset of bipolar disorder coupled with psychotic features in an otherwise healthy, high functioning African American student. The patient, Mr. X also had no reported psychiatric illness in the family. In yet another study, it has been pointed out that one of the risk factors for developing psychotic symptoms in cannabis users is a baseline "psychotic vulnerability"; MMPI data indicate that Mr. X possessed "elements of paranoia, grandiosity, risk of addiction, and antisocial traits."

The authors criticize the current research for failing to elaborate upon the directionality of cannabis use with respect to the occurrence of mental health disorders. The authors recapitulate the available research and formulated the following hypotheses:

\author{
Volume 3 Issue I - 2017 \\ Faisal A Islam, Waquar Siddiqui, Zia \\ Choudhry \\ Department of Mental Health and Clinical Research, Canada
}

Correspondence: Faisal A. Islam, Department of Mental Health and Clinical Research, International Maternal and Child Health Foundation, NPO, Canada, Email islfai@sgu.edu

Received: October 30, 2016 | Published: March 01, 2017

1. Cannabis use precedes the development of psychotic symptoms in healthy individuals

2. Cannabis use facilitates the development of psychotic symptoms in predisposed individuals

The authors have noted that there are currently two case studies that have revealed sustained depersonalization even after discontinuing cannabis use but for most cannabis users the symptoms experienced during drug-free intercessions were more or less non-existent. Furthermore, they assert that first-episode mania due to cannabis abuse may serve as an influential precursor for the development of bipolar disorder. Likewise, the authors provided additional support for their claim by citing a study that states that cannabis use in healthy individuals may portend the development of manic symptoms during follow-up periods. ${ }^{4}$

In general, articles connecting cannabis to affective disorders seem to be somewhat lacking in the literature. Although the role of cannabis is not clearly delineated, the authors of this study have cited epidemiological studies that give credence to the fact that bipolar disorder has the highest rate of substance abuse comorbidity of any axis I disorder. ${ }^{4}$ Despite established DSM-IV and ICD classification systems, a study conducted in Valencia, Spain explores the "possibility of a non-dual diagnosis of cannabis-induced mania, which does not appear in the current international categorical criteria". Both DSM-IV and ICD explicitly deny a particular attribute of cannabis, namely, the ability to evoke mood disorders. ${ }^{5}$ Instead, the aforementioned disorders are classified as "cannabis-related disorders not otherwise specified' in the DSM-IV-TR. ${ }^{1}$ Even more troublesome is the fact that the current literature fails to distinguish the line that exists between cannabis-induced mania and psychotic mania that may accompany cannabis use; 'psychotic-mania' is a feature that is more likely to be associated with the development of schizophrenia, as opposed to bipolar disorder.

Even for individuals that present with a baseline affective and/or psychotic disorder, the role of cannabis with respect to the patients' overall clinical course is not entirely clear. Consider the following study involving Israeli psychiatric inpatients. The study is endorsed by the Grant of the Israel Anti Drug Authority. The authors aimed to compare cannabis users with nonusers. The nonuser group consisted of individuals that had a pre-existing major affective and/ or psychotic disorder but without concomitant drug use. The second 
group comprised of individuals carrying the dual-diagnosis of major affective or psychotic disorders and cannabis use. ${ }^{6}$ It was determined that the severity of manic symptoms in the sample of cannabis users was significantly higher for select subscales. However, cannabis users performed more or less on par with nonusers for the general scale. ${ }^{6}$

Despite the perception of anxiolytic and antidepressive properties of cannabis among self-medicating inpatients, the authors have observed that cannabis use often precedes an exacerbation of psychosis and mania [6]. Furthermore, self-medicated cannabis does not preclude the possibility of cannabis as a 'causal' factor in the development of affective complications; the interrelatedness of cannabis and mania expression appears to be supported by epidemiological studies. ${ }^{\text {? }}$

Dr. Henquet's prospective cohort study in the Netherlands appears to have eliminated the ambiguity that is often associated with research that aims to establish a link between cannabis use and subsequent exacerbation of a bipolar disorder. ${ }^{8}$ It was demonstrated unequivocally that cannabis use at baseline led to an increased risk of manic expression during the 3 year follow-up period of the study. The study was notable for having made a distinction between 'psychotic'mania and mania that developed independently of psychosis. The bidirectional nature of the variables was also ruled out in the study design, allowing for a seemingly 'linear relationship' with causal ramification. ${ }^{8,9}$

Furthermore, in a research dissertation from the University of Massachusetts, quantitative analysis of variables related to cannabis use and affective disorders also yielded the following results:

Individuals that lacked a pre-existing or affective symptomatology, cannabis use corresponded with an

"Increased odds of an incident week of extremely elevated or irritable mood accompanied by at least two manic episode criterion B symptoms"

Self-reported cannabis consumption served as an independent risk factor for "incident bipolar spectrum outcomes within the subpopulations in a nationally representative cohort."

The study also evaluated the role of antisocial attributes in firstdegree relatives. It concluded that for individuals devoid of major depressive symptoms, substance contingence, or antisocial attributes in first degree relatives, past year cannabis use is "associated with an increased risk of manic or hypomanic episodes" as well as an "increased risk for incident bipolar spectrum disorders". ${ }^{10}$

\section{Disorder sleep hygiene, cannabis and bipolar disorder}

Sleep is an important variable that plays a pivotal role in affecting the overall course of bipolar disorder. Research has indicated that an individual's sleep is partly governed by an "endogenous endocannabinoid system". The authors of a recent study have posited that routine cannabis consumption will "down regulate" the endocannabinoids, leading to greatly disordered sleep. In order to test this assumption, the authors performed a study concerning 42 cannabis users and proceeded to evaluate the quality of sleep and consumption patterns for the past year. Qualitative and quantitative assessment techniques strongly suggested a "dose-dependent relationship" for cannabis use and dysfunctional sleep. ${ }^{11}$ A prospective longitudinal study examined nearly 3000 adults over a decade long interval for the onset of bipolar disorder. ${ }^{12}$ An unequivocal relationship was found between preexisting disordered sleep and susceptibility to bipolar disorder. Moreover, high-risk individuals were more likely to exhibit issues pertaining to sleep latency and/or "early morning awakenings". ${ }^{2}$ Habitual cannabis users may be at risk for earlier onset bipolar disorder, especially if they are already experiencing disturbances in sleep.

\section{Conclusion}

Cannabis exacerbation of bipolar disorder appears to be supported by a longitudinal study as well as independent research. It is important to make a distinction between psychotic-mania and cannabis-induced mania. Furthermore, clinicians should be aware of the presence of hypomania that might accompany acute cannabis intoxication. Recent studies strongly suggest the existence of cannabis-induced mood disorders (independent of psychosis) in individuals that previously lacked an underlying bipolar condition. Furthermore, cannabis disturbs the restorative properties of sleep, predisposing habitual users to precipitated onset of bipolar disorder. Parents and clinicians should work in tandem to monitor high-risk individuals for unusual changes in personality and sleep hygiene.

\section{Acknowledgements}

None.

\section{Conflict of interest}

The author declares no conflict of interest.

\section{References}

1. Sadock BJ, Sadock VA. Substance-Related Disorders Kaplan \& Sadock's Concise Textbook of Clinical Psychiatry. Second Edition. 2004. p. 101102.

2. Rottanburg D, Robins AH, Ben Arie O, et al. Cannabis-associated psychosis with hypomanic features. Lancet. 1982;2(8312):1364-1366.

3. Ben AM, Potvin S. Cannabis and psychosis: what is the link? $J$ Psychoactive Drugs. 2007;39(2):131-42.

4. Khan M, Akella S. Cannabis-Induced Bipolar Disorder with Psychotic Features. Psychiatry (Edgmont). 2009;6(12):44- 48.

5. Bertolin Guillen JM, Lopez Arquero FJ, Martinez-Franco L. Cannabisinduced mania? Journal of Substance Use. 2009;13(2):139-141.

6. Katz G, Durst R, Shufman E, et al. Cannabis abuse and severity of psychotic and affective disorders in Israeli psychiatric inpatients. Compr Psychiatry. 2010;51(1):37-41.

7. Richardson TH. A review of the relationship between cannabis use and affective disorders. University of Bath. URJHS. Volume 8.

8. Henquet C, Krabbendam L, De Graaf R, et al. Cannabis use and expression of mania in the general population. $J$ Affect Disord. 2006;95(1-3):103-110.

9. Cannabis use increases risk of developing symptoms of mania. Evidence Based Mental Health. 2007;10(2):61.

10. Mc Cabe PJ. Cannabis use and bipolar disorder: bipolar disorder case identification and cannabis use risk assessment: a dissertation. University of Massachusetts Medical School. 2011.

11. McDaniel K. Cannabis Use and Sleep Quality in Emerging Adults. 2015.

12. Ritter PS, Hofler M, Wittchen HU, et al. Disturbed sleep as risk factor for the subsequent onset of bipolar disorder-Data from a 10-year prospective-longitudinal study among adolescents and young adults. $J$ Psychiatr Res. 2015;68:76-82. 